FERMILAB-Conf-91/282

\title{
The Performance of the Tevatron Collider at Fermilab
}

\author{
N. Gelfand \\ Fermi National Accelerator Laboratory \\ P.O. Box 500, Batavia, Illinois 60510
}

October 1991

* Presented at the Symposium on High-Brightness Beams for Advanced Accelerator Applications, University of Maryland, College Park, July 1991. 


\title{
The Performance of the Tevatron Collider at Fermilab
}

\author{
Norman M. Gelfand \\ Fermi National Accelerator Laboratory, Batavia IL 60510 *
}

July 31,1991

\section{Introduction}

Fermilab is a national laboratory devoted to research in high energy particle physics and is the site of the world's largest proton accelerator. The beams used in the experiments are produced by protons accelerated through a series of accelerators, the last of which is the Tevatron, which raise the energy of the protons from their rest energy of $938 \mathrm{MeV}$ to a final energy of $900 \mathrm{GeV}$ or $1.44 \mathrm{erg}$.

The process begins with a $\mathrm{H}^{-}$jon source input to a $750 \mathrm{keV}$ Cockcroft-Walton electrostatic accelerator. The ions are then accelerated to a kinetic energy of $200 \mathrm{MeV}$ in a drift tube linac. The acceleration, now of protons, continues to a kinetic energy of $8 \mathrm{GeV}$, after charge exchange injection into the Booster, an alternate gradient synchrotron built from combined function magnets, .

The next stage of acceleration occurs in the Main Ring, an alternate gradient synchrotron built with conventional copper-iron magnets arranged in a separated function lattice. At an energy of $150 \mathrm{GeV}$ the protons in the Main Ring are extracted and injected into the Tevatron. It too is an alternate gradient synchrotron but it is constructed with superconducting magnets operating at liquid helium temperature. In the Tevatron the proton energy is raised to $900 \mathrm{GeV}$.

Because the primary purpose of Fermilab is to perform research in high energy physics, the requirements of the research program determine, within of course the abjlity of the accelerators to satisfy those requirements, the significant parameters of the beam at Fermilab, viz the energy, intensity and emittance. From the perspective of the accelerator physicist, the performance of the accelerator may not be optimized; to an experimenter, a particular set of operating conditions may be optimal. The operating conditions of the Tevatron are set to satisfy the experimenter.

- Operated by Universities Research Association Inc. under contract with the U.S. Department of Energy 
This paper will describe the actual operating performance of the Tevatron, operating as a collider, and will indicate the planned upgrades which will enhance the physics results coming from the experiments being performed at Fermilab.

\section{From Source to Injection Into the Tevatron.}

\section{From the Source through the Linac}

The acceleration process begins with a $\mathrm{H}^{-}$source. The properties of the Fermilab $\mathrm{H}^{-}$source are summarized in Table I.

Table I.

Properties of the Source

\begin{tabular}{|l|c|}
\hline Current & $\approx 50 \mathrm{~mA}$ \\
Pulse Length & $30 \mu \mathrm{s}$ \\
Repetjtion Rate & $15 \mathrm{~Hz}$ \\
Number of Ions/sec & $\left(\approx 1.4 \cdot 10^{14} \mathrm{H}^{-}\right.$jons $\left./ \mathrm{s}\right)$. \\
\hline
\end{tabular}

Upon exiting from the source the kinetic energy of the $\mathrm{H}^{-}$ions is raised to $750 \mathrm{keV}$ by a Cockcroft-Walton electrostatic accelerator. They are then transported to and injected into a drift tube linac which increases the kinetic energy to $200 \mathrm{MeV}$.

At the exit from the Cockcroft-Walton, we can make our first measurement of the area of the beam in phase space. These measurements are destructive and are not routinely done; they are made only for diagnostic purposes. The area $A$ of the beam in the phase space $x x^{\prime}$ or $y y^{\prime}$ is called an emittance $\epsilon$. It must be recognized however that the particles are not normally confined within a well defined area in the phase space. This leads to the unfortunate situation where there are several definitions of the emittance defined in terms of $\sigma$ and $\sigma^{\prime}$, the rms widths of the measured spatial and angular distributions. At Fermilab, we have defined the emittance, assuming that the distribution in phase space is Gaussian, in terms of the area that would include $95 \%$ of the particles. As a result we calculate the emittance as $\varepsilon=6 \pi \cdot \sigma \sigma^{\prime}$. Other high energy physics laboratories use other definitions for the emittance: at CERN, $\epsilon=4 \pi \cdot \sigma \sigma^{\prime}$ while at the SSC, $\epsilon=\pi \cdot \sigma \sigma^{\prime}$.

Table II gives the emittance of the beam at the exit from the Cockcroft-Walton at a kinetic energy of $750 \mathrm{keV}$, at the entrance to the linac and at the exit from the linac, where the $\mathrm{H}^{-}$ions have a kinetic energy of $200 \mathrm{MeV}$. 
Table II

Emittance of the $\mathbf{H}^{-}$Ions $^{1}$

\begin{tabular}{|l|c|c|c|}
\hline Location & Kinetjc Energy & $\begin{array}{c}\epsilon(h) \\
(\mathrm{mmmr})\end{array}$ & $\begin{array}{c}\epsilon(v) \\
(\mathrm{mmmr})\end{array}$ \\
\hline $\begin{array}{l}\text { Exit from the } \\
\text { Cockcroft-Walton }\end{array}$ & $750 \mathrm{keV}$ & $\approx 25 \pi$ & $\approx 50 \pi$ \\
\hline Entrance to the Linac & $750 \mathrm{keV}$ & $\approx 68 \pi$ & $\approx 75 \pi$ \\
\hline Exit from the Linac & $200 \mathrm{MeV}$ & $\approx 7.7 \pi$ & $\approx 8.0 \pi$ \\
\hline
\end{tabular}

There is shrinkage of the emittance in the linac but that is just an artifact of the acceleration process: the longitudinal momentum has increased while the transverse momentum is not changed. Consequently $q^{\prime}$, and the area in phase space are reduced. To better characterize what is happening to the beam we define the normalized emittance:

$$
\epsilon_{n}=\epsilon \cdot \beta_{r} \cdot \gamma_{\tau}
$$

where $\beta_{r}=v / c$ and $\gamma_{r}=E / m_{p} c^{2}$.

Table IIl gives the value of the normalized emittances of the beam from the exit of the Cockcroft-Walton to the end of the linac. The normalized emittance increases by almost a factor of two in the linac.

Table III

\begin{tabular}{|l|c|c|c|}
\multicolumn{4}{c}{ Normalized Emittance of the $\mathbf{H}^{-}$Ions $^{1}$} \\
\hline & Kinetic Energy & $\begin{array}{c}\epsilon_{n}(h) \\
(\mathrm{mmmr})\end{array}$ & $\begin{array}{c}\epsilon_{n}(v) \\
(\mathrm{mmmr})\end{array}$ \\
\hline $\begin{array}{l}\text { Exit from the } \\
\text { Cockcroft-Walton }\end{array}$ & $750 \mathrm{keV}$ & $1.0 \pi$ & $2.0 \pi$ \\
\hline Entrance to the Linac & $750 \mathrm{keV}$ & $2.7 \pi$ & $3.0 \pi$ \\
\hline Exit from the Linac & $200 \mathrm{MeV}$ & $5.3 \pi$ & $5.5 \pi$ \\
\hline
\end{tabular}

\section{The Booster}

Upon entering the booster a thin foil is used to strip the two electrons from the $\mathrm{H}^{-}$ion yjelding a bare proton. (The process is $98-99 \%$ efficient.) The protons are then captured by the magnetic field of the Booster.

The Booster is a rapid cycling $(15 \mathrm{~Hz})$ alternate gradient synchrotron which raises the proton kinetic energy to $8 \mathrm{GeV}$. The emittance of the beam in the Booster depends on the number of protons being accelerated. Table IV gives the values of the emittance at the end of the Booster cycle.

\footnotetext{
${ }^{1}$ These data are from an internal Fermilab report EXP-111 The Beam Emittance by Sho Ohnuma, Nov. 28,1983
} 


\section{Normalized Emittance at the Exit From the Booster}

\section{- Kinetic Energy $8 \mathrm{GeV}$}

$\mathrm{N}$ is the number of protons in $10^{10}$ per Booster bunch

- $\epsilon_{n}(h)=\left(4.03+2.03 N-0.911 \mathrm{~N}^{2}\right) \pi$

- $\epsilon_{n}(v)=\left(5.86+1.18 \mathrm{~N}+0.766 \mathrm{~N}^{2}\right) \pi$

\section{The Main Ring}

From the Booster, the $8 \mathrm{GeV}$ protons are transported to the Main Ring where the energy is raised to $150 \mathrm{GeV}$ total energy. The Main Ring, for the data that I will discuss, was operating in the collider mode which is different from the mode of operation for the fixed target experiments. When operating in the collider mode the protons are coalesced into a bunch before they are extracted from the Main Ring and injected into the Tevatron.

In the Main Ring emittance measurements are not destructive and are made with flying wires located in the accelerator. The wires measure the beam profile, and with a knowledge of the lattice parameters at the location of the wires, the emittances can be computed. Table $V$ gives the emittances of the beam at various times in the Main Ring cycle. The data are from measurements made on the beam during the last collider running period and are not the results of special measurements made during study periods. The data were taken under a variety of beam intensities and are characteristic of the emittances actually encountered during the run.

Table $V$

Normalized Emittance in the Main Ring

\begin{tabular}{|l|c|c|}
\hline $\begin{array}{l}\text { Time in the } \\
\text { Main Ring Cycle }\end{array}$ & $\begin{array}{c}\epsilon_{n}(h) \\
(\mathrm{mmmr})\end{array}$ & $\begin{array}{c}\epsilon_{n}(v) \\
(\mathrm{mmmr})\end{array}$ \\
\hline Injection $(8 \mathrm{GeV})$ & $\approx 10 \pi$ & $\approx 12 \pi$ \\
\hline Flat Top $(150 \mathrm{GeV})$ & $\approx 10-15 \pi$ & $\approx 10-15 \pi$ \\
\hline Coalesced Beam $(150 \mathrm{GeV})$ & $\approx 15-20 \pi$ & $\approx 15-20 \pi$ \\
\hline
\end{tabular}

\section{The Tevatron}

\section{The Accelerator}

The Tevatron is a large (radius $=1 \mathrm{~km}$, the same as the Main Ring) proton accelerator constructed from superconducting magnets. It was designed to accelerate protons to an energy of $1 \mathrm{TeV}$. It is currently operating at an energy of $900 \mathrm{GeV}$. 
In the near future, by lowering the temperature of the superconducting magnets by $\approx 0.4 \mathrm{~K}$, we will be able to raise the energy to $1 \mathrm{TeV}$.

In terms of its lattice, the Tevatron is a strong focussing, separated function accelerator. In addition to the arcs, containing the bending dipoles and the focussing quadrupoles, there are 6 long straight sections located in the lattice. Of these, one is used for injecting the beams, another is used for the r.f. accelerating cavities and a third was used as an interaction region for $p \bar{p}$ collisions.

The orbits of protons and anti-protons in the Tevatron were the same and so they could easily be made to collide. ${ }^{2}$ The problem in building the collider was to create a source of anti-protons with high brightness ${ }^{3}$ and to preserve the brightness of the protons and anti-protons as they are accelerated and as they pass from one accelerator to another. ${ }^{4}$

In the collider mode, a bunch of coalesced protons from the main ring was injected into the Tevatron and stored there at $150 \mathrm{GeV}$. This process was repeated 6 times so that there were 6 bunches of protons stored in the Tevatron at $150 \mathrm{GeV}$. For reasons to be discussed later the emittance of the protons was then deliberately increased.

Following the injection the 6 bunches into the Tevatron, anti-protons were removed from the anti-proton source, injected into the Main Ring, accelerated to $150 \mathrm{GeV}$, coalesced into an anti-proton bunch and injected into the Tevatron. This too was repeated 6 times.

The 6 proton bunches and the 6 anti-proton bunches were then accelerated to $900 \mathrm{GeV}$. The lattice was then modified to increase the probability of collisions at the interaction point containing the CDF detector. The beam was also scraped horizontally to eliminate the tails of the particle distribution and to reduce the background in the detector.

As in the Main Ring, the beam emittance measurements can be made using a flying wire system to measure the beam profile. Table VI gives the emittances of the beam at various times in the Tevatron cycle. Again the data are from measurements made on the beam during the last collider running period and are characteristic of the emittances actually encountered. The measurements at low- $\beta$ are made after the beam has been scrapped to reduce background in the experimental detector.

\footnotetext{
${ }^{2}$ In the next collider run the proton and anti-proton orbits will be different due to electrostatic separators inserted into the Tevatron latice.

${ }^{3}$ I will not discuss the problems associated with producing the anti-protons and creating a high brightness source.

${ }^{4}$ The preservation of the brightness during the transfer of the beam from one accelerator to another, becomes increasingly difficult as the energy of the beam increases.
} 
Table VI

Normalized Emittance in the Tevatron

\begin{tabular}{|c|c|c|}
\hline $\begin{array}{l}\text { Time in the } \\
\text { Tevatron Cycle }\end{array}$ & $\begin{array}{c}\epsilon_{n}(h) \\
(\mathrm{mmmr})\end{array}$ & $\begin{array}{c}\epsilon_{n}(v) \\
(\mathrm{mmmr})\end{array}$ \\
\hline Injection $(150 \mathrm{GeV})$ & $\approx 15-20 \pi$ & $\approx 20-25 \pi$ \\
\hline Before Acceleration ( & $\approx 18-25 \pi$ & $\approx 20-28 \pi$ \\
\hline Flat Top $(900 \mathrm{GeV})$ & $\approx 20-25 \pi$ & $\approx 25-30 \pi$ \\
\hline Low $\beta(900 \mathrm{GeV})$ & $\approx 15-20 \pi$ & $\approx 20-25 \pi$ \\
\hline
\end{tabular}

The energy of the collisions (the energy in the Center of Mass system) was $1.8 \mathrm{TeV}$. The collision of a $900 \mathrm{GeV}$ proton with a proton at rest has an equivalent center of mass energy of $41 \mathrm{GeV}$, so that the advantage of a collider in achieving high collision energy is obvious.

\section{Luminosity}

The reason for building the Tevatron was to provide high energy beams for the study of elementary particle collisions. We naturally wish to maximize the number of collisions in a given running period. This objective has a large part in determining the characteristics of the Tevatron beam, and therefore, it is useful to begin our discussion of the characteristics with an expression for the interaction rate $\mathcal{R}$ (interactions/sec) for any reaction:

$$
\mathcal{R}=\sigma \cdot \mathcal{L}
$$

where:

$\sigma$ is the cross section for a given reaction $\left(\mathrm{cm}^{2}\right)$;

$\mathcal{L}$ is the flux or luminosity of the collider $\left(\left(\mathrm{cm}^{-2} \cdot \mathrm{sec}^{-1}\right)\right)$.

The cross section for a typical reaction of interest is $\approx 10^{-33} \mathrm{~cm}^{2}$ or $1 \mathrm{nb}$. If we say that we can collide particles for $10^{7} \mathrm{sec} /$ year $(\approx 30 \%$ efficiency) then we will get $10^{-26} \cdot \mathcal{L}$ jnteractions per year. If we want a sample of $10^{5}$ events to analyze (a typical desire) from a year's run, we will need a luminosity $\mathcal{L}$ of at least $10^{31} /\left(\mathrm{cm}^{2} \cdot \mathrm{sec}\right)$

The luminosity can be expressed in terms of the properties of the colliding proton and anti-proton bunches in the Tevatron. For each colliding bunch:

$$
\mathcal{L}_{\text {bunch }}=\mathcal{F}\left(\sigma_{l}\right) \cdot f_{r} \cdot \frac{N_{p} \cdot N_{\bar{p}}}{\mathcal{A}}
$$

where:

$$
\mathcal{A}=4 \pi \cdot\left(\sigma_{h}(p)^{2}+\sigma_{h}(\bar{p})^{2}\right)^{1 / 2} \cdot\left(\sigma_{v}(p)^{2}+\sigma_{v}(\bar{p})^{2}\right)^{1 / 2}
$$

and where:

- $\mathcal{F}$ is a function of the bunch lengths.

- $f_{\mathrm{r}}$ is the revolution frequency of the beam. 
- $N_{p}$ is the proton bunch intensity.

- $N_{\bar{p}}$ is the anti-proton bunch intensity.

- $\sigma_{h, v}(p),(\bar{p})$ is the rms size of the bunch at the interaction point.

The total luminosity, $\mathcal{L}$, is gotten by summing the luminosities of the colliding bunches, i.e. $\mathcal{L}=\Sigma \mathcal{L}_{\text {bunch }}$.

$\mathcal{A}$ is the effective area of overlap between the proton and anti-proton beams.

The way to increase the luminosity is clear; we increase $N_{p}$ and $N_{\bar{p}}$ and decrease $\mathcal{A}$. This is in fact what is done but only up to a point. To explain why, I will need to make a digression into the Courant-Snyder Theory of the Alternate Gradient Synchrotron.

The equation for the transverse motion for a single particle in a synchrotron can be written as

$$
q^{\prime \prime}+k(s)^{2} q=F(s, q)
$$

where $q$ is a transverse variable of the particle (horizontal or vertical) and $s$ is a coordinate along the closed orbit of the particle. $k(s)$ is the focussing force due to the quadrupoles in the lattice. Since we have an accelerator of circumference $\mathrm{C}$; $k(s+C)=k(s)$. In the case where $F(s, q)=0.0$ i.e. a linear machine the solution to the equation of motion is

$$
\begin{gathered}
q(s)=a \cdot \beta(s)^{1 / 2} \cdot \cos \left(\phi(s)-\phi_{0}\right) \\
\phi(s)=\phi_{0}+\int_{0}^{s} d s / \beta(s)
\end{gathered}
$$

The function $\beta(s)$ is called the amplitude function and can be made to satisfy the equation $\beta(s+C)=\beta(s)$. Note that $q(s+C) \neq q(s)$ unless $\phi(s+C)=\phi(s)+2 n \pi$ where $\mathrm{n}$ is an integer. If $\mathrm{n}$ is an integer however, the motion is unstable. We define the tune of a beam $\nu=[\phi(s+C)-\phi(s)] /(2 \pi)$. If the tune is an integer, we have a resonance and unstable motion.

There is an invariant of the motion, called the emittance, which is simply the area in the phase space, so we can use the same symbol as before;

$$
\epsilon / \pi=\gamma(s) \cdot q(s)^{2}+2 \alpha(s) \cdot \beta(s) \cdot q q^{\prime}+\beta(s) \cdot q^{2}
$$

where:

$\alpha=-1 / 2 \cdot d \beta / d s ;$ and

$\gamma=\left(1+\alpha^{2}\right) / \beta$.

It is easy to show that the value for $a$ in the expression for $q(s)$ is simply $a=(\epsilon / \pi)^{1 / 2}$ so that

$$
q(s)=\sqrt{\epsilon / \pi \cdot \beta(s)} \cdot \cos \left(\phi(s)-\phi_{0}\right)
$$

The beam in the Tevatron is composed of many particles each with its own value of $\epsilon$. Nonetheless it is possible to relate the size, $\sigma$, of a beam of particles at 
a point in the lattice, with a characteristic value for the emittance for the beam. The relation is very simple:

$$
6 \pi \sigma^{2}(s)=\epsilon \cdot \beta(s)
$$

(I am ignoring the dispersion of the lattice at the location of the flying wire used to measure $\sigma$, and the momentum spread of the beam.)

To decrease $\mathcal{A} \approx \pi \sigma_{x} \cdot \sigma_{y}$ we can decrease the value of $\beta$ at the interaction point and we can decrease the size of the beam by decreasing the emittance $\epsilon$.

The smallest possible value of $\beta$ at the interaction point is typically limited by the strength and aperture of the quadrupoles that focus the beam, and the length of the interaction region, i.e. the size of the detector that is designed to observe the interactions.

The other way of reducing $\mathcal{A}$ would be to reduce the emittance of the beam. Unfortunately we cannot simply reduce the emittance indefinitely because of the effect of the beam-beam forces on the luminosity.

\section{Beam Beam Forces}

The bunches of protons and anti-protons interact via electro-magnetic forces. One result of these forces is to modify the tunes of the beams. In a real accelerator, like the Tevatron, where there is motion in both transverse planes with coupling between them, we can have resonances not only when the tunes are integers but also when the tunes satisfy the following equation:

$$
n_{x} \nu_{x}+n_{y} \nu_{y}=m
$$

where $n_{x}, n_{y} m$ are integers. If, due to the beam-beam force, the tune is shifted to a value which is resonant, then particles will be lost.

For the anti-protons the linear beam-beam tune shift at each crossing is given by

$$
\delta \nu \approx \frac{N_{p} \cdot r_{p} \cdot \beta}{4 \pi \cdot \gamma_{r} \cdot \mathcal{A}_{p}}
$$

. In this expression

- $r_{p}$ is the classical radius of the proton $\left(1.53510^{-18} \mathrm{~m}\right)$;

- $\beta$ is the value of the amplitude function at the crossing point.

- $\gamma_{r}$ is the relativistic $\gamma_{r}=\mathrm{E} / \mathrm{m}_{p}$.

- $\mathcal{A}_{p}$ is the cross sectional area of the proton beam at the crossing point. $(\approx \beta \cdot \epsilon)$ 
If the tune shift for the anti-protons is ${ }^{5}$ larger than the distance between the tune of the anti-proton beam and the nearest resonance then we will lose the anti-protons. At the Tevatron, the accelerator was operated with a tune located between the $2 / 5$ resonances and the $3 / 7$ resonances. (During the next collider run the operating tune will be moved so that it lies between the $3 / 5$ and the $4 / 7$ resonances). The available tune space of 0.028 limits the allowed brightness of the protons.

A further consequence of the beam-beam interaction is due to the non-linear part of the interaction. The linear part of the interaction results in the tune shift mentioned above. The non- linear part causes the emittance of the anti-proton beam to blow up. This increase in the emittance reduces the luminosity of the Tevatron collider.

We have found that the maximum integrated luminosity over the length of a store $(\approx 20 h r)$ is achieved when the beam-beam interactions are reduced by increasing the emittance of the protons over their emittance at injection.

\section{Past Experience}

In the past, collisions at the detector began after the protons and anti-protons had been injected in to the Tevatron, accelerated to an energy of $900 \mathrm{GeV}$ and the lattice modified so that the value of $\beta$ at the interaction point was $\approx 0.5 \mathrm{~m}$. The protons and anti-protons were typically stored and collisions observed by the experimenters for $\approx 20 \mathrm{hr}$ and then the store was intentionally ended.

The luminosity was monitored by detectors located around the interaction point. The measured luminosity showed an initially rapid fall (short lifetime) followed by a more gradual decline after about $5 \mathrm{hr}$. The decrease in luminosity was not due to the decrease in the number of protons or anti-protons in the Tevatron but rather it was due to the increase in the emittance of the beams. The increase in emittance was due, in roughly equal parts we think, to intra-beam interactions, beam gas interactions in the relatively poor vacuum in the warm regions of the Tevatron, and to some electrical noise in one or more devices. The length of the poor vacuum regions will be reduced in the next collider run since the separator insertions will have much better vacuum then the previous insertions. We have not identified the sources of the electrical noise so that they are a little hard to fix.

\section{Modifications to Improve Collider Performance.}

Fermilab is currently involved in a program designed to increase the luminosity of the Tevatron collider.

\footnotetext{
${ }^{5}$ The tune shift of the protons is smaller than that of the anti-protons and is not relevant to the following discussion.
} 


\section{Collider Run Ia (1991-1992).}

The beam-beam interactions are among the most important factors limiting the Juminosity of the Tevatron. We have been running with six bunches of protons and six bunches of anti-protons in the Tevatron. During one revolution of the accelerator each bunch will collide 2 times with another bunch, for a total of 12 collisions. Each of these collisions results in a beam-beam tune shift $\delta \nu$ but only at the locations of the detectors do the collisions yield any physics. The number of collisions points, and hence the beam-beam effects, can be reduced if the proton and anti-proton beams are separated except at the interaction points where the detectors are located. We will use electro-static separators inserted into the Tevatron lattice to produce the helical orbits which will result in the separation of the proton and anti-proton orbits. We are planning two low $\beta$ interaction regions in this run so that the number of places where the beambeam interactions take place will be reduced from 12 to 2 by using the separators. This will allow us to reduce the emittance of the protons. We can reduce it by only $40 \%$ due to the limitations in the Booster and Main Ring. In addition we will be making improvements in the anti-proton source to increase the number of anti-protons in each bunch.

Together with a small reduction in $\beta$ at the interaction point, we expect the nominal luminosity to increase from $1.6 \cdot 10^{30} / \mathrm{cm}^{2}$ sec to $5.7 \cdot 10^{30} / \mathrm{cm}^{2} \mathrm{sec}$, an improvement by a factor of $\approx 3.6$.

\section{Collider Run Ib.}

The next step in our improvement schedule will center on increasing the production rate of anti-protons. This will require beams of higher brightness in the Main Ring since the aperture of the Main Ring currently limits the intensity that can be accelerated. The brightness of the beam in the Main Ring is in turn limited by the brightness of the Booster beam. In the Booster the brightness is limited by the intra-beam tune shift at injection due to space charge. The space charge tune shift can be reduced by increasing the energy of the Linac beam.

We currently have underway a linac upgrade program, to be completed in 1992 , which will increase the kinetic energy of the protons from $200 \mathrm{MeV}$ to $400 \mathrm{MeV}$. This will result in a increased anti-proton stacking rate and a luminosity of $9.0 \cdot 10^{30} / \mathrm{cm}^{2} \mathrm{sec}$, an improvement by a factor of $\approx 5.6$ from our last run.

\section{Collider Run II.}

In the subsequent run we expect to run at our original design energy of $1 \mathrm{TeV}$. In addition we will have installed new kickers for the injection of anti-protons and so we will be able to inject 36 bunches of protons and anti-protons. Neither the proton bunch intensities nor the emittances will differ from the previous run. At 
this point the luminosity will be limited by the available number of anti-protons. We expect that due to the higher energy the larger number of bunches and the smaller beam-beam effects that the luminosity will rise to $1.1 \cdot 10^{31} / \mathrm{cm}^{2} \mathrm{sec}$, an improvement by a factor of $\approx 6.9$ from our last run.

\section{Collider Run III.}

To significantly increase the number of anti-protons available for the Tevatron, and thereby the luminosity, it will be necessary to replace the Main Ring by a new Main Injector. It will then be possible to optimize the intensity and emittances of the bunches to achieve the maximum luminosity. With the new injector the Tevatron collider, which was designed to have a luminosity of $10^{30}$, will achieve a luminosity of $5.7 \cdot 10^{31} / \mathrm{cm}^{2} \mathrm{sec}$, an improvement by a factor of $\approx 36$ from our last run.

The improvements are summarized in Table VII.

Table VII

Projected Luminosity Improvements In the Tevatron Collider ${ }^{6}$

\begin{tabular}{|l|c|c|c|c|}
\hline Running Period & I & la & II & III \\
\hline Center of Mass Energy (Gev) & $88-89$ & 1991 & 1992,1994 & 1996 \\
Number of Bunches & 1800 & 1800 & 2000 & 2000 \\
Protons/bunch $\left(10^{10}\right)$ & 6 & 6 & 36 & 36 \\
Anti-protons/bunch $\left(10^{10}\right)$ & 7 & 7 & 12 & 33 \\
Total Antj-protons $\left(10^{10}\right)$ & 2.9 & 7.2 & 1.2 & 3.7 \\
Anti-proton Stacking Rate $\left(10^{10} / \mathrm{hr}\right)$ & 2 & 43 & 43 & 130 \\
$\epsilon_{p}(\mathrm{mmmr})$ & $25 \pi$ & $15 \pi$ & $15 \pi$ & $30 \pi$ \\
$\epsilon_{\bar{p}}(\mathrm{mmmr})$ & $18 \pi$ & $18 \pi$ & $18 \pi$ & $22 \pi$ \\
$\beta^{*}(\mathrm{~cm})$ & 55 & 50 & 50 & 50 \\
Luminosity $\left(10^{30} \mathrm{~cm}^{-2} \cdot \mathrm{sec}^{-1}\right)$ & 1.6 & 5.7 & 11 & 57 \\
\hline
\end{tabular}

With this new luminosity the Tevatron at Fermilab will be doing exciting physics experiments well into the $21^{\text {st }}$ century.

\section{Acknowledgements}

It is a please to acknowledge the very helpful assistance of David Finley, Karl Koepke and Charles Schmidt in the preparation of this paper.

\footnotetext{
${ }^{6}$ S. Holmes 1991 IEEE-PAC
} 